\title{
CRITÉRIOS PARA AVALIAÇÃo DA MORFOLOGIA DO GRÃO DE AVEIA PARA O MELHORAMENTO GENÉTICO DA QUALIDADE FÍSICA
}

\author{
CRITERIA TO EVALUATE GRAIN MORPHOLOGY FOR OAT QUALITY BREEDING
}

\author{
Cristhiane Regina Abegg Bothona ${ }^{1}$ Sandra Cristina Kothe Milach ${ }^{2}$ Gladis Hermes Thomé ${ }^{3}$ \\ Cândida Braga Cabral ${ }^{4}$ Luis Marcelo Tisian ${ }^{5}$ Gislaine Ososki Mellos ${ }^{5}$
}

\section{RESUMO}

Apesar da morfologia do grão ser de interesse no melhoramento da aveia, critérios de amostragem $e$ análise de grãos para a seleção da qualidade física neste cereal precisam ser determinados. Os objetivos deste trabalho foram determinar as correlações entre características morfológicas de grãos primários, secundários e terciários de aveia, com e sem casca, a fim de definir de que forma podem ser manipuladas no melhoramento genético dessa espécie. Cinqüenta grãos primários, secundários e terciários, com e sem casca, de dez genótipos de aveia foram avaliados para área, largura, comprimento, perímetro e fator de formato através da análise de imagens digitais. Variabilidade entre genótipos de aveia foi encontrada para todas as características. As correlações entre as características de morfologia de grãos primários, secundários e terciários, em grãos com e sem casca, foram altas e significativas. Assim, a determinação do tamanho do grão de um genótipo pode ser feita pela avaliação da área de grãos primários com casca; enquanto que medidas de comprimento $e$ largura são necessárias para definir o tipo característico de grão do genótipo. Baixas correlações foram obtidas entre as características de grãos terciários com os demais, e a eliminação destes pode contribuir para um aumento da uniformidade e maior qualidade física de grãos em aveia.

Palavras-chave: comprimento, largura, uniformidade de grãos.

\section{SUMMARY}

Although kernel morphology is of great interest in oat breeding, it is still necessary to determine how to proceed and analyze samples in selecting for better physical kernel quality in this cereal. The objectives of this study were to determine the correlation among primary, secondary and tertiary kernel morphology traits to define strategies for breeding these traits in oat. Fifty primary, secondary and tertiary, hulled and dehulled kernels from ten oat genotypes were analyzed for area, length, width, perimeter and shape factor through digital image analysis.
Variability among genotypes was identified for all kernel morphology traits. High and significant correlation was found among traits evaluated in primary, secondary, tertiary, hulled and dehulled kernels. Therefore, the average kernel size of a genotype can be determine by the area of its primary hulled kernels; and the evaluations of kernel width and length are necessary for determining kernel shape. Low correlation was found between tertiary kernels and the other two types and selecting against this may contribute for increasing physical kernel quality in oat.

Key words: Avena sativa, grain width and length, grain uniformity, DIA.

\section{INTRODUÇÃO}

O crescente uso de grãos e derivados de aveia na alimentação humana, aliado à procura de qualidade pelo mercado consumidor, têm feito com que os melhoristas selecionem genótipos com qualidade superior de grãos.

A qualidade de grãos em aveia depende de vários fatores, que podem estar relacionados a aspectos químicos ou físicos do grão. Para a definição de qualidade física do grão em aveia, o peso de hectolitro e a relação grão/casca têm sido indicadores clássicos (FORSBERG \& REEVES, 1992). Esses, contudo, são de difícil seleção em gerações segregantes iniciais, como F2 e F3, porque sua análise requer uma quantidade mínima de sementes não disponível de plantas individuais. Dessa forma, a identificação de características de grão que podem ser analisadas em amostras de

\footnotetext{
${ }^{1}$ Biólogo, Mestre em Fitotecnia, Novartis, Uberlândia, MG.

${ }^{2}$ Engenheiro Agrônomo, PhD., Professor do Departamento de Plantas de Lavoura, Faculdade de Agronomia (FA), Universidade Federal do Rio Grande do Sul (UFRGS). Av. Bento Gonçalves, 7712, CP 776, 91501-970, Porto Alegre, RS. Autor para correspondência.

${ }^{3}$ Biólogo, Mestre em Botânica, aluno do Programa de Pós-graduação em Fitotecnia, FA/UFRGS.

${ }^{4}$ Engenheiro Agrônomo, aluno do Programa de Pós-graduação em Fitotecnia, FA/UFRGS.

${ }^{5}$ Alunos do Curso de Agronomia, UFRGS, bolsistas Iniciação Científica, PIBIC/CNPq e FAPERGS.
} 
plantas individuais e estejam relacionadas com o peso de hectolitro $(\mathrm{PH})$ e a relação grão/casca em aveia, tem sido de grande interesse. Alguns estudos relatam que aspectos da morfologia do grão em aveia influenciam o peso hectolitro de grãos e as características de rendimento industrial (FORSBERG \& REEEVES, 1992; BOTHONA, 1997). Assim, a identificação de genótipos de aveia com tamanho e forma de grão superiores pode auxiliar no melhoramento para rendimento industrial nessa espécie.

A análise da morfologia dos grãos até pouco tempo não podia ser feita rotineiramente em programas de melhoramento de aveia devido às dificuldades de efetuá-la. Essas decorriam do fato de os métodos disponíveis, baseados no uso de paquímetros (FLOSS et al., 1996) ou da projeção de sombras das sementes sobre papel milimetrado (MURPHY \& FREY, 1962), serem laboriosos e pouco precisos para avaliar um grande número de amostras.

Com o desenvolvimento da análise de imagens digitalizadas (AID) para a determinação precisa de tamanho e forma de órgãos e estruturas vegetais (SYMONS \& FULCHER, 1988a e b; PIETRZAK \& FULCHER, 1995; SAPIRSTEIN, 1995; FULCHER et al., 1996; THOMÉ et al., 1997), a avaliação da morfologia do grão de aveia em um grande número de amostras passou a ser viável. Dessa forma, características morfológicas de grão podem ser avaliadas em amostras com pequeno número de grãos obtidos de uma planta.

A obtenção rápida e precisa de medidas de grão, através de AID, possibilita o estudo da uniformidade de tamanho de grãos em genótipos de aveia, característica de importância no beneficiamento industrial desta espécie (BOTHONA, 1997). Também a relação grão/casca influencia o rendimento industrial deste cereal e pode ser estudada através da análise de imagens de grãos com e sem casca. A existência de grãos primários, secundários e terciários na inflorescência da aveia, e a possibilidade de AID ser efetuada tanto em grãos com como sem casca, fazem com que haja a necessidade de se estabelecer critérios de avaliação da morfologia do grão nessa espécie. Para tanto, as correlações entre medidas da morfologia dos diferentes tipos de grãos em aveia devem ser estabelecidas. Altas correlações entre medidas obtidas do grãos primários, secundários e terciários e grãos com e sem casca podem determinar que avaliações em um tipo de grão seriam suficientes para estabelecer o padrão de qualidade física do grão de um genótipo de aveia. Isso facilitaria a automatização da AID na rotina de programas de melhoramento desse cereal. Assim, os objetivos deste trabalho foram determinar as correlações entre características morfológicas de grãos primários, secundários e terciários de aveia, com e sem casca, a fim de definir de que forma podem ser manipulados no melhoramento genético desta espécie.

\section{MATERIAL E MÉTODOS}

Foram utilizados dez genótipos de aveia provenientes de programas de melhoramento do Sul do Brasil, sendo eles: UFRGS 7, UFRGS 8, UFRGS 14, UFRGS 15, UFRGS 17, UFRGS 18; UFRGS 911747, CTC 3, CTC 6 e UPF 16.

$\mathrm{O}$ experimento foi conduzido na Estação Experimental Agronômica da UFRGS (EEA/UFRGS) em Eldorado do Sul - RS, no ano de 1996, em um delineamento experimental de blocos completamente casualizados com três repetições. As parcelas foram constituídas de cinco linhas com cinco metros de comprimento, com espaçamento de 0,20 metros entre linhas, e densidade de semeadura igual a 300 sementes aptas $/ \mathrm{m}^{2}$. Os tratos culturais foram realizados conforme recomendação técnica para o cultivo da aveia.

Após a maturação, em cada parcela foi coletada a panícula do colmo principal de cinco plantas, que foram mantidas em câmara fria a $10{ }^{\circ} \mathrm{C}$ na EEA/ UFRGS, até o momento da análise. Após debulha das panículas, foram obtidas, aleatoriamente, amostras de 50 grãos primários, 50 grãos secundários e, quando presentes, grãos terciários, até o máximo de 50. Essas amostras foram mantidas em câmara fria até serem submetidas à análise de imagens digitalizadas para obtenção de dados das seguintes características morfológicas de grão para cada genótipo, em grãos com casca e após descascados: área $\left(\mathrm{em} \mathrm{mm}^{2}\right)$; comprimento, largura e perímetro $(\mathrm{mm})$ e fator de formato (varia de zero a um, sendo zero para uma linha e um para um círculo perfeito). As imagens de grão foram captadas através de vídeo-câmera, e transferidas para computador através de uma placa de vídeo externa. Os grãos de cada amostra foram dispostos com o vinco voltado para baixo, para proporcionar homogeneidade na captação das imagens, as quais foram analisadas com o programa SIGMA SCAN / IMAGE 1.0. Um quadrado de papel de tamanho padronizado $\left(1 \mathrm{~cm}^{2}\right)$ presente em todas as imagens foi utilizado para a conversão dos dados de "pixels" para milímetros.

Os dados obtidos foram submetidos à análise de correlação fenotípica para as características medidas em grãos primários, secundários e terciários, com e sem casca. 


\section{RESULTADOS E DISCUSSÃO}

Grande variabilidade foi observada entre genótipos para todas as características de grãos avaliadas com e sem casca. Na tabela 1, são apresentados os valores médios de tamanho e formato de grão para amostras contendo mistura de grãos primários, secundários e terciários. Esses descrevem o tipo característico de grão obtido em um lote de sementes de cada genótipo.

Altas e significativas correlações foram encontradas entre as características de morfologia de grãos primários, secundários e terciários, tanto em grãos com casca como sem casca (tabela 2). As correlações em grãos sem casca foram em geral mais altas do que aquelas obtidas em grãos com casca. As maiores diferenças foram observadas naquelas que envolveram comprimento do grão (tabela 2). De fato, o comprimento é a medida mais alterada quando comparadas as avaliações de grãos com e sem casca (tabela 3).

A área apresentou correlação com comprimento, largura e perímetro do grão com e sem casca (tabela 2). Dessa forma, pode ser considerada uma característica que representa, de forma geral, o tamanho do grão. Contudo, a área não está correlacionada com o fator de formato, tendo em vista que este componente expressa a forma do grão e não o tamanho. Essas relações entre componentes foram observados por DE KOYER $\boldsymbol{e t}$ al. (1993), que relataram aumento para área, comprimento, largura e perímetro de grão ao longo de ciclos de seleção para rendimento de grãos em aveia, enquanto que $o$ formato dos grãos permaneceu constante. O perímetro e o comprimento de grão apresentaram correlações significativas em grãos com e sem casca (tabela 2). Essa alta correlação e a ausência de correlação entre largura e perímetro indica que o perímetro é definido basicamente pelo comprimento do grão.

A largura e o comprimento do grão não se apresentaram correlacionados, indicando que são características independentes (tabela 2). Estes resultados coincidem com os obtidos por DE KOYER et al. (1993) e MURPHY \& FREY (1962), e podem estar relacionados a fatores de desenvolvimento do grão, conforme descrito por MURPHY \& FREY (1962) e BORÉM (1997). Segundo estes autores, enquanto o comprimento do grão é definido até o oitavo dia do desenvolvimento do grão, a largura é definida ao longo de toda a fase de maturação, pois esta característica é dependente do enchimento do grão.

Também foram observadas correlações entre componentes de qualidade física do grão com e sem casca, indicando que o tamanho do grão com casca reflete o seu tamanho após o descasque (tabela 3). Contudo, o comprimento foi a única característica em que esta correlação não foi significativa. Este resultado pode estar relacionado ao fato de o comprimento do grão ser determinado na panícula anteriormente ao enchimento do grão, e

Tabela 1 - Médias da mistura de grãos primários, secundários e terciários para área, comprimento, largura, perímetro e fator de formato de grãos com (cc) e sem (sc) casca de dez genótipos de aveia. F.A./UFRGS, 1996.

\begin{tabular}{|c|c|c|c|c|c|c|c|c|c|c|}
\hline \multirow{2}{*}{ Genótipos } & \multicolumn{2}{|c|}{ área $\left(\mathrm{mm}^{2}\right)$} & \multicolumn{2}{|c|}{ comprimento (mm) } & \multicolumn{2}{|c|}{ largura (mm) } & \multicolumn{2}{|c|}{ perímetro (mm) } & \multicolumn{2}{|c|}{ fator de formato } \\
\hline & $\mathrm{cc}$ & $\mathrm{sc}$ & $\mathrm{cc}$ & $\mathrm{sc}$ & $\mathrm{cc}$ & $\mathrm{sc}$ & $\mathrm{cc}$ & $\mathrm{sc}$ & $\mathrm{cc}$ & $\mathrm{sc}$ \\
\hline UFRGS 7 & $26,1 \quad \mathrm{D}$ & $16,4 \mathrm{C}$ & $13,0 \mathrm{BCD}$ & $8,4 \mathrm{CDE}$ & $2,5 \quad \mathrm{~F}$ & $2,4 \quad \mathrm{~F}$ & $29,7 \mathrm{CD}$ & $19,6 \mathrm{DE}$ & $0,36 \quad E$ & $0,52 \mathrm{D}$ \\
\hline UFRGS 8 & $30,6 \mathrm{AB}$ & $21,1 \mathrm{~A}$ & $13,3 \mathrm{BC}$ & $9,0 \mathrm{~B}$ & $3,1 \mathrm{~B}$ & $2,9 \mathrm{AB}$ & $30,0 \mathrm{BCD}$ & $21,5 \mathrm{BC}$ & $0,42 \mathrm{AB}$ & $0,55 \mathrm{C}$ \\
\hline UFRGS 14 & $30,6 \mathrm{AB}$ & $20,1 \mathrm{~A}$ & $13,9 \mathrm{~B}$ & $8,7 \mathrm{BCD}$ & $3,0 \mathrm{BC}$ & 2,8 BCD & $31,3 \mathrm{BC}$ & $20,7 \mathrm{CD}$ & $0,40 \mathrm{BCD}$ & $0,56 \mathrm{BC}$ \\
\hline UFRGS 15 & $29,5 \mathrm{BC}$ & $19,3 \mathrm{AB}$ & $13,4 \mathrm{~B}$ & $8,4 \mathrm{CDE}$ & $3,0 \mathrm{BC}$ & 2,8 BCD & $30,4 \mathrm{BC}$ & $20,0 \mathrm{DE}$ & $0,39 \mathrm{D}$ & $0,58 \mathrm{AB}$ \\
\hline UFRGS 17 & $32,3 \mathrm{~A}$ & $20,8 \mathrm{~A}$ & $14,9 \mathrm{~A}$ & $9,8 \mathrm{~A}$ & $2,9 \mathrm{C}$ & $2,6 \quad \mathrm{E}$ & $31,4 \mathrm{~A}$ & $23,0 \mathrm{~A}$ & $0,36 \quad E$ & $0,49 \quad E$ \\
\hline UFRGS 18 & $28,2 \mathrm{CD}$ & $17,4 \mathrm{BC}$ & $12,5 \mathrm{CD}$ & $8,0 \quad E$ & $3,0 \mathrm{BC}$ & $2,7 \quad \mathrm{DE}$ & $28,4 \mathrm{DE}$ & $19,3 \quad E$ & $0,43 \mathrm{~A}$ & $0,57 \mathrm{C}$ \\
\hline UFRGS 911747 & $32,6 \mathrm{~A}$ & $21,1 \mathrm{~A}$ & $13,6 \mathrm{~B}$ & $8,4 \mathrm{CDE}$ & $3,2 \mathrm{~A}$ & $3,0 \mathrm{~A}$ & $31,4 \mathrm{~B}$ & $20,5 \mathrm{CDE}$ & $0,40 \mathrm{CD}$ & $0,60 \mathrm{~A}$ \\
\hline CTC 3 & $26,3 \mathrm{D}$ & $17,7 \mathrm{BC}$ & $13,2 \mathrm{BC}$ & $8,7 \mathrm{BC}$ & $2,6 \quad \mathrm{E}$ & $2,4 \quad F$ & $29,8 \mathrm{BCD}$ & $20,5 \mathrm{CDE}$ & $0,36 \quad \mathrm{E}$ & $0,51 \mathrm{D}$ \\
\hline CTC 5 & $26,9 \quad \mathrm{D}$ & $18,1 \mathrm{BC}$ & $12,2 \mathrm{D}$ & $8,2 \mathrm{DE}$ & $2,8 \quad \mathrm{D}$ & 2,8 BCD & $27,8 \quad \mathrm{E}$ & $19,6 \mathrm{DE}$ & $0,42 \mathrm{ABC}$ & $0,58 \mathrm{BC}$ \\
\hline UPF 16 & $28,3 \mathrm{CD}$ & $21,2 \mathrm{~A}$ & $13,7 \mathrm{~B}$ & $9,6 \mathrm{~A}$ & $2,6 \quad \mathrm{E}$ & $2,7 \quad \mathrm{DE}$ & $31,3 \mathrm{BC}$ & $22,5 \mathrm{AB}$ & $0,36 \quad \mathrm{E}$ & $0,51 \quad \mathrm{D}$ \\
\hline $\mathrm{CV}$ & 4,0 & 5,3 & 3,5 & 3,2 & 1,8 & 3,0 & 2,9 & 3,0 & 3,2 & 2,2 \\
\hline
\end{tabular}

Médias seguidas de mesma letra na coluna não diferem significativamente ao teste Duncan a 5\%.

Ciência Rural, v. 29, n. 4, 1999. 
Tabela 2 - Coeficientes de correlação fenotípica entre área, comprimento, largura, perímetro e fator de formato de grãos de aveia com (cc) e sem (sc) casca ${ }^{\dagger}$. F.A./UFRGS, 1996

\begin{tabular}{|c|c|c|c|c|c|c|c|c|}
\hline & \multicolumn{2}{|c|}{ área } & \multicolumn{2}{|c|}{ comprimento } & \multicolumn{2}{|c|}{ largura } & \multicolumn{2}{|c|}{ perímetro } \\
\hline & $\mathrm{cc}$ & $\mathrm{sc}$ & $\mathrm{cc}$ & $\mathrm{sc}$ & $\mathrm{cc}$ & $\mathrm{sc}$ & $\mathrm{cc}$ & $\mathrm{sc}$ \\
\hline comprimento & $0,43^{*}$ & $0,70 * *$ & & & & & & \\
\hline largura & $0,68 * *$ & $0,68 * *$ & 0,06 & $-0,01$ & & & & \\
\hline perímetro & $0,74 * *$ & $0,80 * *$ & $0,63 * *$ & $0,98 * *$ & 0,25 & 0,15 & & \\
\hline fator formato & 0,12 & $-0,03$ & $-0,31$ & $-0,70 * *$ & $0,50 * *$ & $0,66 *$ & $-0,48 * *$ & $-0,59 * *$ \\
\hline
\end{tabular}

${ }^{\dagger}$ grãos primários, secundários e terciários foram utilizados na análise.

**significativo a $1 \%$.

* significativo a $5 \%$.

dessa forma, mesmo um grão curto pode apresentar lema e pálea longas (MURPHY \& FREY, 1962).

Um aspecto muito importante é a uniformidade do tamanho de grãos de aveia em uma amostra onde estão misturados grãos primários, secundários e terciários, pois é desta forma que os grãos de aveia chegam à indústria para o beneficiamento. Quanto mais próximo for o tamanho destes grãos, maior é a uniformidade, o que garante à indústria um maior rendimento industrial. As correlações das medidas de morfologia do grão entre grãos primários e secundários, primários e terciários e secundários e terciários, apresentados nas tabelas 4 a 6, revelam como são estas relações em grãos com e sem casca. A diagonal dessas tabelas, destacada em negrito, indica que as correlações mais altas são entre grãos primários e secundários (tabelas 4 a 6). Os grãos terciários tendem a ser bem menores que os primários e secundários (BOTHONA, 1997) e sua

Tabela 3 - Coeficientes de correlação fenotípica entre componentes de qualidade física de grãos de aveia com (cc) e sem (sc) casca ${ }^{\dagger}$. F.A./ UFRGS, 1996.

\begin{tabular}{llclll}
\hline & $\begin{array}{c}\text { área } \\
\mathrm{cc}\end{array}$ & $\begin{array}{c}\text { comprimento } \\
\mathrm{cc}\end{array}$ & $\begin{array}{c}\text { largura } \\
\mathrm{cc}\end{array}$ & $\begin{array}{c}\text { perímetro } \\
\mathrm{cc}\end{array}$ & $\begin{array}{c}\text { formato } \\
\mathrm{cc}\end{array}$ \\
\hline & & & & & \\
& & & & & \\
área sc & $0,70 * *$ & 0,36 & $0,59 * *$ & $0,46 *$ & 0,19 \\
comprimento sc & 0,23 & 0,35 & 0,06 & 0,35 & $-0,22$ \\
largura sc & $0,46 *$ & $-0,05$ & $0,75 * *$ & 0,06 & $0,55 * *$ \\
perímetro sc & $0,62 * *$ & $0,70 * *$ & 0,12 & $0,71 * *$ & $-0,37$ \\
formato sc & $-0,05$ & $-0,53 * *$ & $0,53 * *$ & $-0,04 *$ & $0,43 * *$ \\
& & & & & \\
\hline
\end{tabular}

† grãos primários, secundários e terciários foram utilizados na análise.

**significativo a $1 \%$.

* significativo a $5 \%$. ocorrência é dependente de fatores ambientais que alteram o suprimento de reservas para o enchimento dos grãos (SYMONS \& FULCHER, 1996). A ausência de grãos terciários em alguns genótipos utilizados neste estudo, bem como a diferença de tamanhos destes em comparação aos grãos primários e secundários, levaram a baixas correlações entre grãos terciários e os demais (tabelas 5 e 6 ).

As correlações entre medidas da morfologia de grãos obtidas individualmente para grãos primários, secundários e terciários foram semelhantes àquelas obtidas para uma amostra com a presença dos três tipos de grãos (tabela 2), apesar das correlações envolvendo grãos terciários tenderem a ser mais baixas (tabelas 5 e 6), devido à ausência destes em alguns genótipos. As baixas correlações entre grãos terciários com os outros tipos de grãos indicam que a seleção contra grãos terciários pode levar à obtenção de genótipos com maior uniformidade para tamanho de grão.

De forma geral, pode-se afirmar que a área é uma medida que indica o tamanho do grão, estando relacionada ao peso de grão, característica de grande importância para o melhoramento de aveia (SYMONS \& FULCHER, 1988; DE KOYER et al., 1993; BOTHONA, 1997). Contudo, esta característica por si só não representa o formato do grão. Assim, para determinar o padrão de qualidade física de um genótipo, ou seja, se este apresenta grãos longos ou curtos, largos ou finos, dados de comprimento e largura do grão devem ser obtidos. 
Tabela 4 - Coeficientes de correlação fenotípica entre área, comprimento, largura, perímetro e fator de formato de grãos primários e secundários de aveia com e sem casca. F.A./ UFRGS, 1996.

\begin{tabular}{|c|c|c|c|c|c|c|c|c|c|c|}
\hline primários & \multicolumn{2}{|c|}{ área } & \multicolumn{2}{|c|}{ comprimento } & \multicolumn{2}{|c|}{ largura } & \multicolumn{2}{|c|}{ perímetro } & \multicolumn{2}{|c|}{ formato } \\
\hline secundários & $\mathrm{cc}$ & $\mathrm{sc}$ & $\mathrm{cc}$ & $\mathrm{sc}$ & $\mathrm{cc}$ & $\mathrm{sc}$ & $\mathrm{cc}$ & $\mathrm{sc}$ & $\mathrm{cc}$ & $\mathrm{sc}$ \\
\hline área & $\mathbf{0 , 6 0} * *$ & $\mathbf{0 , 8 1} * *$ & $0,57 * *$ & $0,64 * *$ & $0,53 * *$ & $0,50 * *$ & $0,60 * *$ & 0,74 & $-0,04$ & 0,01 \\
\hline comprimento & $0,51 * *$ & $0,60 * *$ & $0,68 * *$ & $\mathbf{0 , 8 5} * *$ & 0,35 & 0,09 & $0,68 * *$ & $0,85 * *$ & $-0,38$ & $-0,40$ \\
\hline largura & $0,58 * *$ & $0,76 * *$ & 0,31 & 0,25 & $\mathbf{0 , 6 7} * *$ & $\mathbf{0 , 7 3} * *$ & 0,37 & 0,41 & 0,39 & 0,41 \\
\hline perímetro & $0,53 * *$ & $0,67 * *$ & $0,68 * *$ & $0,83 * *$ & 0,38 & 0,20 & $\mathbf{0 , 6 8} * *$ & $\mathbf{0 , 8 5} * *$ & $-0,34$ & $-0,31$ \\
\hline formato & $-0,04$ & 0,19 & $-0,39$ & $-0,51$ & 0,15 & $0,61 * *$ & $-0,36$ & $-0,36$ & $0,65 * *$ & $\mathbf{0 , 7 5} * *$ \\
\hline
\end{tabular}

**significativo a $1 \%$

* significativo a 55 .

Tabela 5 - Coeficientes de correlação fenotípica entre área, comprimento, largura, perímetro e fator de formato de grãos primários e terciários de aveia com e sem casca. F.A./ UFRGS, 1996.

\begin{tabular}{|c|c|c|c|c|c|c|c|c|c|c|}
\hline primários & \multicolumn{2}{|c|}{ área } & \multicolumn{2}{|c|}{ comprimento } & \multicolumn{2}{|c|}{ largura } & \multicolumn{2}{|c|}{ perímetro } & \multicolumn{2}{|c|}{ formato } \\
\hline terciários & $\mathrm{cc}$ & $\mathrm{sc}$ & $\mathrm{cc}$ & $\mathrm{sc}$ & $\mathrm{cc}$ & $\mathrm{sc}$ & $\mathrm{cc}$ & $\mathrm{sc}$ & $\mathrm{cc}$ & $\mathrm{sc}$ \\
\hline área & $\mathbf{0 , 2 0}$ & 0,24 & 0,28 & 0,18 & 0,17 & 0,16 & 0,27 & 0,21 & $-0,05$ & 0,01 \\
\hline comprimento & 0,03 & 0,08 & $\mathbf{0 , 3 0}$ & $0,60 * *$ & $-0,08$ & $-0,36$ & 0,26 & 0,51 & $-0,36$ & $-0,61 * *$ \\
\hline largura & 0,29 & 0,29 & 0,14 & $-0,01$ & $\mathbf{0 , 3 9}$ & $\mathbf{0 , 3 7}$ & 0,17 & 0,07 & 0,26 & 0,25 \\
\hline perímetro & 0,04 & 0,12 & 0,29 & 0,52 & $-0,06$ & $-0,24$ & 0,25 & 0,46 & $-0,33$ & $-0,48$ \\
\hline formato & 0,24 & 0,23 & $-0,01$ & $-0,41$ & 0,36 & $0,61 * *$ & 0,02 & $-0,27$ & 0,43 & $0,66 * *$ \\
\hline
\end{tabular}

**significativo a $1 \%$.

$*$ significativo a $5 \%$.

Tabela 6 - Coeficientes de correlação fenotípica entre área, comprimento, largura, perímetro e fator de formato de grãos secundários e terciários de aveia com e sem casca. F.A./ UFRGS, 1996.

\begin{tabular}{|c|c|c|c|c|c|c|c|c|c|c|}
\hline secundários & \multicolumn{2}{|c|}{ área } & \multicolumn{2}{|c|}{ comprimento } & \multicolumn{2}{|c|}{ largura } & \multicolumn{2}{|c|}{ perímetro } & \multicolumn{2}{|c|}{ formato } \\
\hline terciários & $\mathrm{cc}$ & $\mathrm{sc}$ & $\mathrm{cc}$ & $\mathrm{sc}$ & $\mathrm{cc}$ & $\mathrm{sc}$ & $\mathrm{cc}$ & $\mathrm{sc}$ & $\mathrm{cc}$ & $\mathrm{sc}$ \\
\hline área & $\mathbf{0 , 5 8} * *$ & $\mathbf{0 , 5 0}$ & 0,36 & 0,38 & 0,40 & 0,48 & 0,38 & 0,42 & 0,10 & 0,21 \\
\hline comprimento & 0,36 & 0,36 & $\mathbf{0 , 3 3}$ & $0,61 * *$ & 0,10 & 0,06 & 0,33 & $0,56 *$ & $-0,11$ & $-0,37$ \\
\hline largura & $0,60 * *$ & 0,46 & 0,25 & 0,20 & $0,56 * *$ & $\mathbf{0 , 5 6} * *$ & 0,33 & 0,27 & 0,32 & 0,41 \\
\hline perímetro & 0,39 & 0,43 & 0,31 & $0,58 *$ & 0,13 & 0,19 & $\mathbf{0 , 3 1}$ & $0,56 *$ & $-0,07$ & $-0,22$ \\
\hline formato & 0,33 & 0,22 & 0,05 & $-0,22$ & 0,45 & 0,52 & 0,10 & $-0,12$ & 0,28 & $\mathbf{0 , 6 9} * *$ \\
\hline
\end{tabular}

**significativo a $1 \%$

* significativo a $5 \%$.

Ciência Rural, v. 29, n. 4, 1999. 


\section{CONCLUSÃO}

O tamanho do grão de um genótipo de aveia pode ser determinado pela avaliação da área do grão com casca, em uma amostra com a presença dos diferentes tipos de grãos (primários, secundários e terciários); As medidas de comprimento e largura são necessárias para determinar o formato e o tipo específico de grão de um genótipo. As avaliações de grãos sem casca são necessárias apenas para uma precisa determinação do comprimento do grão. O tamanho de grãos primários reflete aquele dos grãos secundários de uma panícula, mas a medida de ambos os tipos é necessária para identificação de genótipos com maior uniformidade de grãos.

\section{AGRADECIMENTOS}

Os autores agradecem o apoio financeiro da FAPERGS, CNPq e CAPES que possibilitou a realização deste trabalho.

\section{REFERÊNCIAS BIBLIOGRÁFICAS}

BORÉM, A. Melhoramento de plantas. Viçosa: UFV, 1997. 547 $\mathrm{p}$

BOTHONA, C.R.A. Qualidade do grão de aveia: características físicas e regiões genômicas associadas. Porto alegre - RS, 1997. 97 p. Dissertação (Mestrado em Fitotecnia) - Faculdade de Agronomia, Universidade Federal do Rio Grande do Sul, 1997.

DE KOYER, D.L., STUTHMAN, D.D., FULCHER, R.G. $\boldsymbol{e} \boldsymbol{t}$ al Effects of recurrent selection for grain yield on oat kernel morphology. Crop Science, Madison, v. 33, n. 2, p. 924-928, 1993
FLOSS, E.L., SCHULZ, J., TRENTIN, E. A. Qualidade industrial de grãos de cultivares de aveia em Passo Fundo, 1994. In REUNIÃO DA COMISSÃO SULBRASILEIRA DE PESQUISA DA AVEIA, 16., 1996, Florianópolis. Anais.... Florianópolis: UFSC, 1996.547 p. p. 143-148.

FORSBERG, R.A., REEVES, D.L. Breeding oat cultivars for improved grain quality. In: MARSHALL, M.G., SORRELS M.E. Oat science and technology. Madison: Crop Science Society America Inc. Publishers, 1992. P. 756-775.

FULCHER, R.G., CHURCHILL, K., MEDIN, T. Image analysis for cereal improvement. In: INTERNATIONAL OAT CONFERENCE, 5.; INTERNATIONAL BARLEY GENETICS SYMPOSIUM, 7., 1996, Saskatoon. Proceedings... Saskatoon: University of Saskatchewan, 1996, v. 1,485 p. p. $73-76$

MURPHY, C.F., FREY, K.J. Inheritance and heritability of seed weight and its components in oats. Crop Science, Madison, v. 2, n. 1 , p. $509-512,1962$

PIETRZAK, L.N., FULCHER,R.G. Polymorphism of oat kernel size and shape in several canadian oat cultivars . Canadian Journal of Plant Science, Ottawa, v. 75, p. 105-109, 1995.

SAPIRSTEIN, H.D. Variety identification by digital image analysis. In: WRIGLEY,C.W. Identification of food-grain varieties. Sydney: Aus. Am. Assoc. of Cereal Chem., 1995. p. $91-130$

SYMONS, S.J., FULCHER, R.G. Determination of variation in oat kernel morphology by digital analysis. Journal of Cereal Science., London, v. 7, n. 1, p. 219-228, 1988a.

SYMONS, S.J., FULCHER, R.G. Determination of wheat kernel morphological variation by digital image analysis: II. Variation in cultivars of soft white winter wheats analysis. Journal of Cereal Science., London, v. 8, n. 1, p. 219-229, 1988b.

THOMÉ,G.C.H, MILACH, S.C.K., BOTHONA, C.R.A., et al. Image analysis for quantitative assessment of oat crown rust Fitopatologia Brasileira, Brasília, v. 22, n. 4, p. 518-520, 1997.

Ciência Rural, v. 29, n. 4, 1999. 